\title{
Una metodología Sistémica y creativa para la gestión estratégica: Caso de Estudio Región de Atacama-Chile
}

\author{
A Systematic and Creative Methodology to Drive Strategic Management: \\ Chilean \\ Case Study in Atacama-Region.
}

Christopher-Nikulin', Gabriela-Becker²

\begin{abstract}
En el marco de la complejidad del análisis estratégico como un proceso que requiere de capacidad analítica, técnica, conocimiento e intuición la creación de una metodología que permita obtener nuevo conocimiento es relevante para anticipar potenciales direcciones de desarrollo. Bajo esta perspectiva, en este artículo se propone una metodología creativa y sistémica para la generación de estrategias en distintos contextos basándose en herramientas tradicionales de estrategia y herramientas creativas como es la teoría de resolución de problemas (TRIZ). Finalmente, un caso de estudio real es desarrollado con el objetivo de mostrar la aplicabilidad de la metodología en una industria Chilena relevante, como es la industria minera.

A strategic analysis can be considered as a complex process that requires specific analytical skills as technique, knowledge and intuition. A new methodology needs to be capable of obtaining new knowledge to anticipate a more holistic strategic directions. With this perspective, in this article is proposed a creative and systematic methodology to develop strategic analysis considering different context, the methodology is based on traditional tools from strategic literature and creative tools from problem solving literature known as TRIZ. Moreover, the methodology was conceived as step by step structure in order to support beginners for the application of this type of analysis. Finally, an interesting and real case study was developed in order to show the applicability of the methodology in a relevant industry of Chile as the mining industry.
\end{abstract}

Keywords: metodología; estrategia; minería; teoría de resolución de problemas de inventiva; methodology; strategy; mining; theory of problem solving (triz)

\footnotetext{
1,2Universidad Técnica Federico Santa María,Av. España 1680,Valparaíso-Chile. e-mail: 'christopher.nikulin@usm.cl, ${ }^{2}$ gabriela.becker@alumnos.usm.cl
}

ISSN: 07 I8-2724. (http://www.jotmi.org) 


\section{Introducción}

Uno de los rasgos indiscutibles del entorno mundial es el aumento del riesgo y de la incertidumbre como resultado de la constante transformación de la sociedad, de la abrumadora cantidad y velocidad de los cambios sociales, y de su influencia sobre las organizaciones, los sectores productivos, las regiones y los países (Klinger, 2006). En este contexto, las organizaciones y los tomadores de decisión, deben proveerse de métodos y herramientas de planeamiento estratégico que permitan enfrentar de la mejor forma los cambios y desafíos del entorno en virtud de su desarrollo y alcance de objetivos.

No obstante, el planeamiento estratégico es un proceso complejo (Koontz y Weihrich, 1994) que se basa en la integración de la intuición y el análisis en la toma de decisiones (David, 2003) para la formulación de estrategias que guíen a la organización en conocimiento de sus capacidades y recursos actuales en medio de la incertidumbre del entorno y del futuro. La dificultad para quienes toman las decisiones radica en el manejo, estructuración y análisis de la información relacionada a la organización, a la conceptualización del contexto de la empresa, a la reflexión de las probables consecuencias y a la elección de alternativas adecuadas para los problemas en la práctica. Se requiere entonces de técnicas que faciliten el proceso de análisis y selección de estrategias a través del diseño y empleo de herramientas que conduzcan a la conformidad de las decisiones y delimitación objetiva de las opciones de solución, factibles y deseables (Acevedo et al., 20I0)

En el marco de la complejidad del análisis estratégico como un proceso que requiere de capacidad analítica, técnica e intuitiva, se ha vuelto cada vez más interesante el poder proponer una metodología, estructurada y flexible que logre entregar direcciones y guías en la formación de estrategias más aun para quienes no son expertos, como sucede en el caso de emprendimientos universitarios, start-up o en empresas con poca experiencia. Dado este contexto, en este artículo se propone una metodología sistémica para el análisis estratégico y sus direcciones, siguiendo una serie de pasos estructurados como: Primero, identificación del contexto actual utilizando un modelo creativo de forma de ampliar la dimensión de estudio del analista. Segundo, generación de parámetros relevantes para el estudio a través de herramientas tradicionales de análisis estratégico. Tercero, evaluación cualitativa de la información obtenida y por último, propuesta de direcciones a seguir basado en la teoría de resolución de problemas de inventiva (TRIZ).
Este artículo está estructurado de la siguiente manera: La segunda sección describe el estado del arte para la aplicación de la metodología propuesta, focalizado en los conceptos de análisis estratégico y sus herramientas; FODA, como método tradicional;yTRIZ, como herramienta creativa para estimular la búsqueda de oportunidades. La tercera sección describe la metodología propuesta, su estructura y procedimiento, así como las herramientas útiles para su ejecución, método de análisis y generación de estrategias. Finalmente, la cuarta sección presenta un caso de estudio para validar la metodología en el contexto de la industria minera en Chile en la región de Atacama en el contexto del proyecto Cluster minero que es financiado por la Corporación de Fomento y Desarrollo (CORFO). Finalmente se presentan la discusión, limitaciones y conclusiones de la metodología propuesta así como recomendaciones futuras.

\section{Estado del Arte \\ I.I Análisis estratégico y herramientas}

La Planeación Estratégica es el proceso mediante el cual quienes toman decisiones en una organización obtienen, procesan y analizan información pertinente, interna y externa, con el fin de evaluar la situación presente de la empresa, así como su nivel de competitividad con el propósito de anticipar y decidir sobre el direccionamiento de la institución hacia el futuro (Serna, 1997). Con dicha planeación se busca proyectar a la empresa por medio de un proceso basado en las estrategias (Harris, 2006)

En marco del proceso de planeación y dirección estratégica (David, 2003) se encuentra el análisis estratégico, que consiste en comprender la posición estratégica de la organización en función de su entorno externo, sus recursos y competencias internas, y las expectativas e influencia de los stakeholders (Johnson et al., 2006). El análisis estratégico suele dividirse en el análisis externo o del entorno, que busca identificar oportunidades y amenazas y en el análisis interno de la organización, que determina fortalezas y debilidades. Por consiguiente, el fin del análisis estratégico es conseguir tener una perspectiva de las influencias clave sobre el bienestar presente y futuro de la organización, de las oportunidades que ofrece el entorno, $y$ de las competencias de la organización (Johnson et al., 2006). 


\begin{tabular}{|l|l|l|l|}
\hline Niveles de la Planeación & Fases de Planeación & Herramientas & Resultado \\
\hline \multirow{5}{*}{ Formulación } & Declaración de misión y visión & Diagrama de ABELL & Declaración de misión y visión \\
\cline { 2 - 4 } & Análisis externo & $\begin{array}{l}\text { PESTA } \\
5 \text { fuerzas de PORTER }\end{array}$ & $\begin{array}{l}\text { Identificación de oportunidades y } \\
\text { amenazas }\end{array}$ \\
\cline { 2 - 4 } & \multirow{2}{*}{ Análisis interno } & $\begin{array}{l}\text { Cadena de valor de PORTER; mod- } \\
\text { elo de 7S; ratios financieros; análisis } \\
\text { VRIO }\end{array}$ & $\begin{array}{l}\text { Identificación de debilidades y } \\
\text { fortalezas }\end{array}$ \\
\cline { 2 - 4 } & Formulación de lineamientos & $\begin{array}{l}\text { matriz FODA } \\
\text { matriz PEEA } \\
\text { matriz BCG }\end{array}$ & Determinación de estrategias \\
\hline Implementación & $\begin{array}{l}\text { Formulación de objetivos y pro- } \\
\text { gramas }\end{array}$ & Cuadro de mando integral & Determinación de objetivos \\
\hline Evaluación & Control y medición de resultados & Indicadores de desempeño & Monitoreo y evaluación de impacto \\
\hline
\end{tabular}

Tabla I: Clasificación de los distintos métodos estratégicos y sus resultados.

\section{I.2 Análisis FODA}

Según Dyson (2004), el análisis FODA (también conocida como DOFA, FODA, MAFE en español y SWOT en inglés) es una de las técnicas más empleadas en la planeación estratégica, en especial para la determinación de la posición estratégica de la empresa (Hill, 1997). Por lo demás, es una importante herramienta de apoyo para la toma de decisiones generalmente usada para analizar sistemáticamente los ambientes interno y externo de una organización (Kangas et al., 2003; Kotler, 2000; Stewart et al., 2002).

La herramienta FODA consiste en la construcción de una matriz a partir de la identificación de un listado de factores internos (fortalezas y debilidades) y externos (oportunidades y amenazas) que influyen en el desempeño de la organización. Posteriormente, se contraponen los factores internos con los externos, tal que permita generar estrategias fundamentadas en las fortalezas de la organización para corregir sus debilidades; tomar ventaja de las oportunidades y contrarrestar las amenazas; desarrollando cuatro tipos de estrategias, de acuerdo con lo propuesto por David (2003) como se visualiza en la tabla 2 .

Para Houben (1999), las fortalezas y debilidades conforman un entorno interno que puede ser controlado, mientras que los otros factores están en un ambiente externo que no puede ser manipulado. lbañez et al. (2008), por su parte, señalan que el análisis FODA considera que una estrategia tiene que lograr un equilibrio o ajuste entre la capacidad interna de la organización y su situación de carácter externo (Mintzberg et al. 1997).

\begin{tabular}{|l|l|l|}
\hline & $\begin{array}{l}\text { OPORTUNIDADES } \\
\text { - Cambios en el entorno social, económico, } \\
\text { político, tecnológico } \\
- \text { Nuevas tecnologías y procesos productivos } \\
- \text { Nuevas necesidades del mercado }\end{array}$ & $\begin{array}{l}\text { AMENAZAS } \\
\text { - Resistencia al cambio } \\
\text { - Competitividad } \\
\text { - Altos riesgos y grandes obstáculos }\end{array}$ \\
\hline $\begin{array}{l}\text { FORTALEZAS } \\
\text { - Recursos superiores } \\
\text { - Capacidades distintivas } \\
\text { - Ventajas naturales }\end{array}$ & $\begin{array}{l}\text { Estrategias ofensivas: } \\
\text { Usar las fuerzas para aprovechar las oportuni- } \\
\text { dades }\end{array}$ & $\begin{array}{l}\text { Estrategias defensivas: } \\
\text { Usar las fuerzas para evitar las amenazas }\end{array}$ \\
\hline $\begin{array}{l}\text { DEBILIDADES } \\
\text { - “Talones de Aquiles" } \\
\text { - Desventajas } \\
\text { - Recursos y capacidades escasas }\end{array}$ & $\begin{array}{l}\text { Estrategias Adaptativas: } \\
\text { Superar las debilidades aprovechando las opor- } \\
\text { tunidades }\end{array}$ & $\begin{array}{l}\text { Estrategias de supervivencia: } \\
\text { Reducir la debilidades y evitar las amenazas }\end{array}$ \\
\cline { 2 - 3 }
\end{tabular}

Tabla 2: Matriz FODA (DOFA) para generación de estrategias.

ISSN: 07 I8-2724. (http://www.jotmi.org)

Journal of Technology Management \& Innovation (C) Universidad Alberto Hurtado, Facultad de Economía y Negocios. 


\section{I.3 Teoría de Resolución de Problemas (TRIZ)}

La Teoría de Resolución de Problemas (TRIZ) fue creada por G. Altshuller (1984) basada en la observación de patentes y su evolución a través del tiempo, TRIZ se basa en tres postulados principales: i) Situación inicial, toda solución depende de su estado del arte y entorno; ii) Contradicción, una solución confiable se obtiene resolviendo las contradicciones y/o conflictos entre sus parámetros además de la optimización de los recursos disponibles; iii) Leyes de Evolución, todo producto y/o proceso tiene una evolución paulatina que puede ser entendida a través de patrones.

Actualmente, el método TRIZ ha evolucionado, y no sólo es efectivo en problemas técnicos, sino que da posibilidad de ser usado para problemas de carácter multidisciplinario, lo cual ha dado origen a la teoría del pensamiento poderoso OTSM-TRIZ (Cavallucci \& Khomenko, 2007: Khomenko et al., 2007), esta evolución hace que TRIZ sea integrable a nuevas áreas del conocimiento (Nikulin et al. 2013).

La piedra angular de TRIZ Clásico radica en la formulación de contradicciones; una contradicción (Altshuller et al. 1999) es un enfrentamiento entre dos aspectos conflictivos en un mismo sistema, el que debe vencerse para lograr un mejoramiento sustancial en el mismo (Altshuller, 1984). La formulación de contradicciones es el inicio del proceso resolutivo, el cual permite focalizar los esfuerzos para la obtención de soluciones y búsqueda de oportunidades.

\section{I.3.I.I Obtención de requisitos basados en conceptos de TRIZ}

Becattini \& Cascini (2013) propone un modelo para identificación y obtención de los requisitos capaces de conducir los procesos de diseño. Donde las direcciones de mejoramiento deben estar basadas en el principio de idealidad propuestas por Altshuller (1984), que menciona que un sistema debe aumentar sus funciones útiles, disminuir las funciones perjudiciales y los recursos asociados a éste.

Becattini \& Cascini (2013) desarrolla un modelo para la obtención de los posibles efectos que se producen como consecuencia de las funciones nocivas de tecnologías en el diseño. Tal modelo está configurado por elementos: i) el objeto de la función útil principal; ii) el sistema y subsistemas de la integridad; iii) el entorno externo. Éste consiste en la representación gráfica de las interacciones potencialmente dañinas que se producen por la aparición de impactos nocivos entre parejas de elementos del sistema técnico, el objeto/producto de la función, y el medio ambiente. Sin embargo, es adaptable a que la misma función (y sus efectos) pueden ser considerados como útiles y nocivos, en función de los contextos en que se estudie.

\section{I.3.I.2 Utilización de los principios inventivos en la búsqueda de nuevas estrategias}

La mayoría de las decisiones de gestión en la organización realizadas por los ejecutivos y directores generales aún se basan en la intuición y la experiencia personal, por lo que a menudo complejos contextos son extremadamente simplificados; alternativas son ignoradas; el riesgo no es evaluado correctamente; y recursos, conocimiento y potencialidades no son utilizados para la mejor resolución de problemas en el momento adecuado (Ruchti \& Livotov, 200I). La aplicación de las herramientas de pensamientoTRIZ para la resolución de problemas de inventiva, reemplaza con el proceso de prueba y error en la búsqueda de soluciones y/o oportunidades. Estos elementos, han contribuido a la creciente demanda por parte de tomadores de decisión a utilizar herramientas sistemáticas de pensamiento como los principios de inventiva de TRIZ, para procesar la información y tomar mejores decisiones en la búsqueda de nuevas estrategias.

La aplicación de la metodología TRIZ comienza con presentar el problema particular encontrado como un problema análogo, transformando tal problema específico en un lenguaje analógico basado en parámetros simples de entender (Cavalluci et al. 2007), cuya función es mostrar otros problemas análogos que han sido resueltos en otras instancias. Posteriormente, con los principios de inventiva se genera una solución análoga. Finalmente, se encuentra una solución específica que soluciona el problema específico inicial (Viveros et al., 2013)

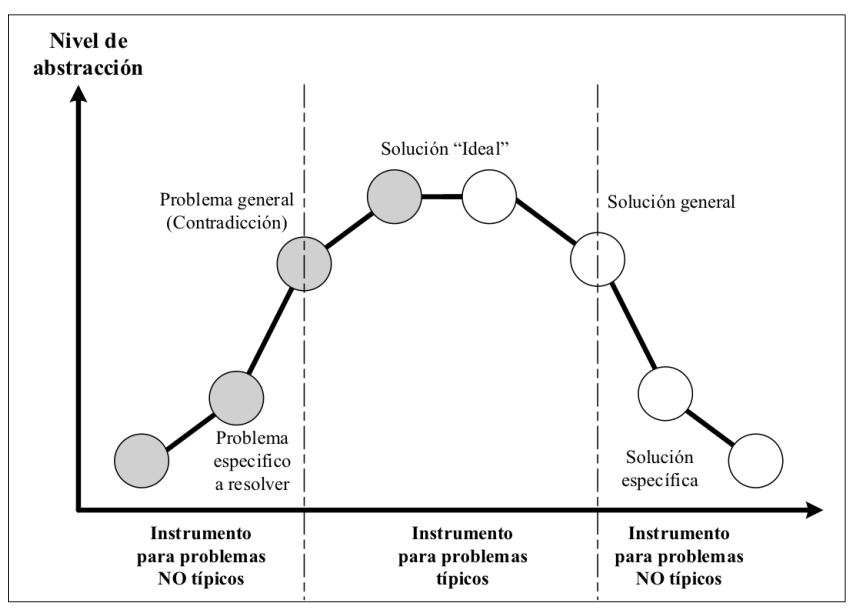

Figura I:Adaptación de esquema de solución de problemas usando TRIZ. 
Este enfoque sistémico presenta ventajas considerables para incrementar la eficiencia del pensamiento creativo además de la factibilidad de las soluciones propuestas (Savransky, 2000).

\section{Metodología propuesta}

Como se ha señalado previamente, se plantea la propuesta de una metodología sistémica para el análisis y formulación de direcciones estratégicas, siguiendo una serie de pasos estructurados y conduciendo su desarrollo desde la obtención de información y generación de estrategia, en la cual se identifican factores relevantes, parámetros críticos y dirección de propuestas estratégicas a través de métodos que estimulen la creatividad y análisis sistémico como son los conceptos de TRIZ.

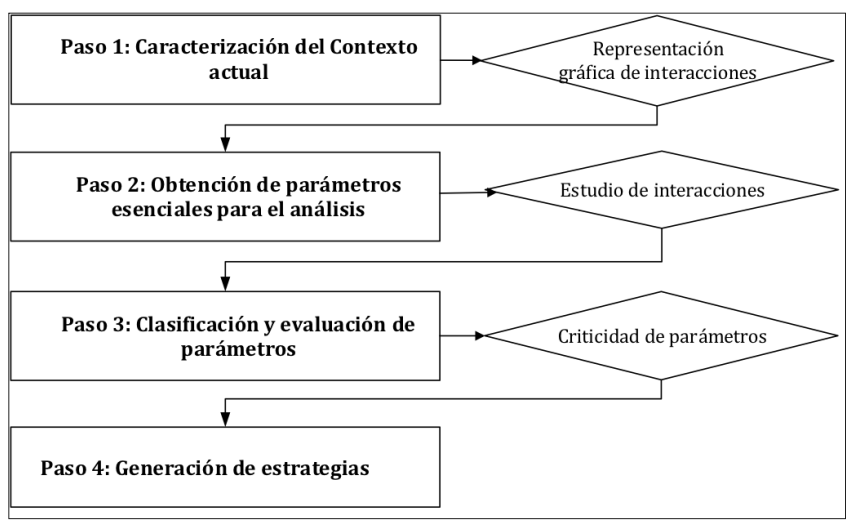

Figura 2: Estructura de pasos de metodología para análisis estratégico.

\section{Paso I: Caracterización del contexto actual}

En el primer paso se busca obtener información sobre el sistema a analizar de manera de proponer una estrategia considerando todos los aspectos relacionado al sistema y el contexto. Dado que el modelo propuesto por Becattini \& Cascini (20/3) es explícitamente para el análisis de un sistema técnico, en este artículo se propone una modificación del modelo con el objetivo de hacerlo adecuado al contexto del análisis estratégico organizacional. La modificación propone un modelo que consiste en la representación gráfica del contexto actual desde una perspectiva sistémica y relacional, donde el objeto a analizar (empresa, organización, conglomerado, clúster u otro) es denominado <sistema>, el cual tiene interacciones con <elementos de su entorno> $y$ desempeña una <función principal $>$ para lograr el alcance de un <objetivo>.
Como muestra la figura 3 , el modelo está constituido por el sistema, los elementos del entorno -pueden ser gobierno, mercado, comunidad, otros-, un objetivo y una función principal. Las flechas grises representan las interacciones positivas (+) y las negras las negativas (-), dirigidas hacia ambos sentidos; tanto desde el sistema hacia los elementos del entorno, como desde cada elemento hacia el sistema, además de las interacciones desde el sistema hacia sí mismo. Adicionalmente, toda interacción del modelo tiene dirección de influencia, que puede ser: i) Sistema>elemento; ii) elemento>sistema; iii) sistema $>$ sistema. $Y$ naturaleza de influencia: i) perjudicial (impacto negativo); y ii) benéfica (impacto positivo), ambas presente en cada relación.

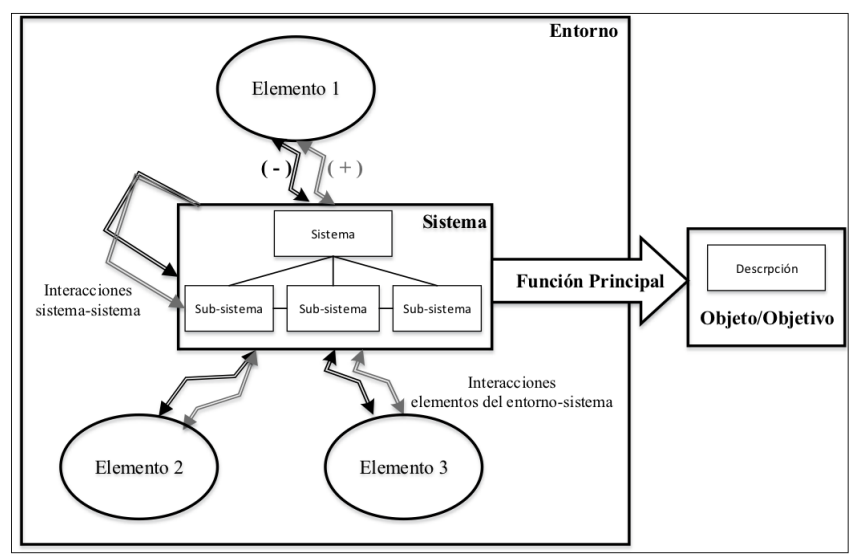

Figura 3 Modelo gráfico de interacciones para análisis estratégico.

\section{I Paso 2: Obtención de parámetros esenciales para el análisis}

Una vez planteada la situación inicial, se deben obtener los parámetros esenciales que describen el contexto actual. Tales parámetros son los factores que influyen de manera positiva, negativa, interna y externa al sistema y su funcionalidad; y que se obtienen a partir de la evaluación de las interacciones existentes entre los elementos. Para cada interacción se estudia la forma en que el elemento influye al sistema y viceversa, positiva y negativamente, a través de las dimensiones de estudio (política, social, económica, competitiva, organizacional, otras) desde la cual se analiza la interacción. Tales dimensiones de estudio son entregadas por la herramienta estratégica que se escoja para obtener la información, como señala la tabla 3. 
Cabe destacar que a pesar de que se entregan orientaciones respecto a la utilización de herramientas para la obtención de parámetros, la elección de la(s) técnicas(s) a utilizar para la evaluación de cada interacción depende exclusivamente del criterio del analista en cuanto al acceso de información, facilidad de dimensiones a utilizar, el enfoque del objetivo u objeto involucrado, el enfoque deseado para las estrategias propuestas, etc.

\subsection{Paso 3: Clasificación y evaluación de parámetros}

Una vez obtenidos los parámetros que sustentan el análisis, éstos se clasifican en fortalezas, oportunidades, debilidades y amenazas según la naturaleza positiva o negativa del impacto del parámetro y la capacidad de control que posea el sistema sobre éste (tabla 4). Posteriormente es necesario asignar a cada uno de los parámetros enlistados, medidas de impacto y de eventualidad con el propósito de determinar su nivel de criticidad para la evaluación de estrategias. Donde Criticidad $=($ Índice nivel de impacto $) \times($ Índice nivel de eventualidad $)$
Tanto la clasificación como la evaluación de parámetros buscan ordenar la información previamente obtenida. El nivel de criticidad del parámetro entrega nociones del grado de importancia o prioridad que se le asigne a éste, determinando el tipo de estrategia a seguir. Para los parámetros negativos, el nivel de criticidad radica en que son parámetros que tienen alto impacto negativo en el sistema y a la vez, una alta probabilidad de ocurrencia, en el caso de amenazas, o alta tasa de frecuencia, en el caso de debilidades. El nivel de criticidad de oportunidades y fortalezas, radica en que son parámetros que tienen alto impacto positivo en el sistema y debido a que poseen una alta probabilidad de ocurrencia (oportunidades) o alta tasa de frecuencia (fortalezas), es vital que se mantengan o potencien, aprovechando tales condiciones para una acción sostenible y duradera, recomendaciones que se presentan más adelante en la matriz de estrategias.

\begin{tabular}{|c|c|c|}
\hline Herramienta & Orientación estratégica & Dimensiones de estudio \\
\hline 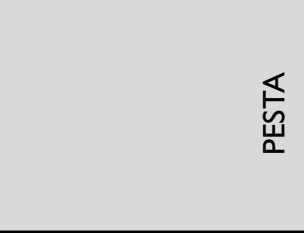 & $\begin{array}{l}\text { Entrega dimensiones de estudio de los ám- } \\
\text { bitos político, económico, social, tecnológico } \\
\text { y ambiental relativos al entorno e influen- } \\
\text { cias sobre la organización. Obtención de } \\
\text { parámetros orientada a la interacción siste- } \\
\text { ma-entorno, objeto-entorno. }\end{array}$ & $\begin{array}{l}\text {-político } \\
\text {-económico } \\
\text {-social } \\
\text {-tecnológico } \\
\text {-ambiental }\end{array}$ \\
\hline 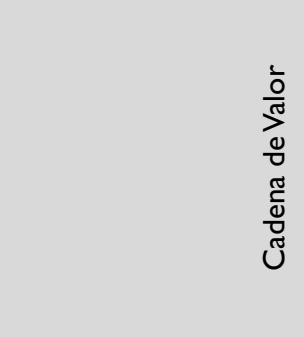 & $\begin{array}{l}\text { Entrega dimensiones de estudio interno del } \\
\text { sistema empresarial o industrial, entregando } \\
\text { información respecto a la estructura de su } \\
\text { cadena de valor productiva. } \\
\text { Obtención de parámetros orientada a in- } \\
\text { teracción sistema-sistema, sistema-objeto, } \\
\text { sistema-entorno en caso de entornos pro- } \\
\text { ductivos. }\end{array}$ & $\begin{array}{l}\text {-infraestructura } \\
\text {-RRHH } \\
\text {-capacidad tecnológica } \\
\text {-adquisiciones } \\
\text {-logística interna } \\
\text {-logística de salida } \\
\text {-operaciones } \\
\text {-comercialización y marketing } \\
\text {-servicios }\end{array}$ \\
\hline
\end{tabular}

Tabla 3: Herramientas para la obtención de parámetros.

\begin{tabular}{|l|c|c|c|}
\hline & & \multicolumn{2}{|c|}{ Impacto del parámetro } \\
\hline & & Negativo & Positivo \\
\hline \multirow{3}{*}{ Capacidad de control } & Interna & DEBILIDAD & FORTALEZA \\
C- & C+ \\
\cline { 2 - 4 } & Externa & AMENAZA & OPORTUNIDAD \\
& & NC+ \\
\hline
\end{tabular}

Tabla 4: Clasificación de parámetros

ISSN: 07I 8-2724. (http://www.jotmi.org) 
La estructura de asignación de niveles de impacto y probabilidad (tabla 5) está basada en la matriz de impacto y probabilidad utilizada frecuentemente en la gestión de riesgos de proyectos, aplicada a los factores externos de la organización con medidas cuantitativas estimadas por los encargados de proyecto en consideración de la naturaleza y características de cada proyecto. La evaluación de la importancia de cada riesgo y, por consiguiente, de su prioridad de atención, se efectúa utilizando una tabla de búsqueda o una matriz de probabilidad e impacto. Según Duncan (1996), dicha matriz especifica las combinaciones de probabilidad e impacto que llevan a calificar los riesgos con una prioridad baja, moderada o alta como se presenta en la tabla 5 .

En este caso, este análisis es aplicado tanto a parámetros internos como externos con índices de evaluación estimados.

\subsection{Paso 4: Generación de estrategias}

Se proponen dos sub-pasos para la generación de estrategias, propuestas en base a los parámetros obtenidos, a través de herramientas matriciales. El paso 4 .I conduce a la selección de estrategias genéricas a través de la matriz creada a partir de elementos de análisis estratégico tradicional, por otra parte, el paso 4.2 conduce a la generación de estrategias específicas a través de la utilización de los principios inventivos de TRIZ y su aplicación a las estrategias generales previamente definidas.

\subsection{Paso 4.I: Matriz de generación de estrategias genéricas}

Esta matriz (tabla 6) entrega direcciones genéricas para los parámetros estudiados, dando nociones del nivel de complejidad o alcance que debe considerar la estrategia a implantar, en virtud del impacto del parámetro sobre el sistema y de la capacidad para controlarlo que éste posea. La construcción de la matriz toma elementos teóricos de la generación de estrategias FODA y de la matriz de impacto y probabilidad de gestión de riesgos de proyectos, basando la generación de estrategias en la entrega de direcciones que anulen o mitiguen los impactos negativos y se aprovechen los efectos positivos, en consideración de la intervención del medio externo y beneficio de externalidades con el propósito de mantener el control del funcionamiento y gestión del sistema. La utilización de la matriz busca orientar a los tomadores de decisión acerca de la priorización de direcciones en la conformación de la estrategia conjunta que guía a la organización o sistema, en base a la criticidad de los elementos presentados por el análisis de parámetros.

\begin{tabular}{|l|l|l|l|}
\hline Nivel de impacto & Índice & Eventualidad & Índice \\
\hline Impacto fundamental & 5 & Seguro (siempre ocurre) & $\mathrm{I}$ \\
\hline Impacto alto & 4 & Muy probable & 0,9 \\
\hline Impacto moderado & 3 & Probable & 0,5 \\
\hline Impacto bajo & 2 & Poco probable & 0,3 \\
\hline Participa pero no impacta & $\mathrm{I}$ & Remoto (nunca ha ocurrido) & 0,1 \\
\hline
\end{tabular}

Tabla 5:Asignación de niveles de impacto y eventualidad

ISSN: 07 I8-2724. (http://www.jotmi.org) 


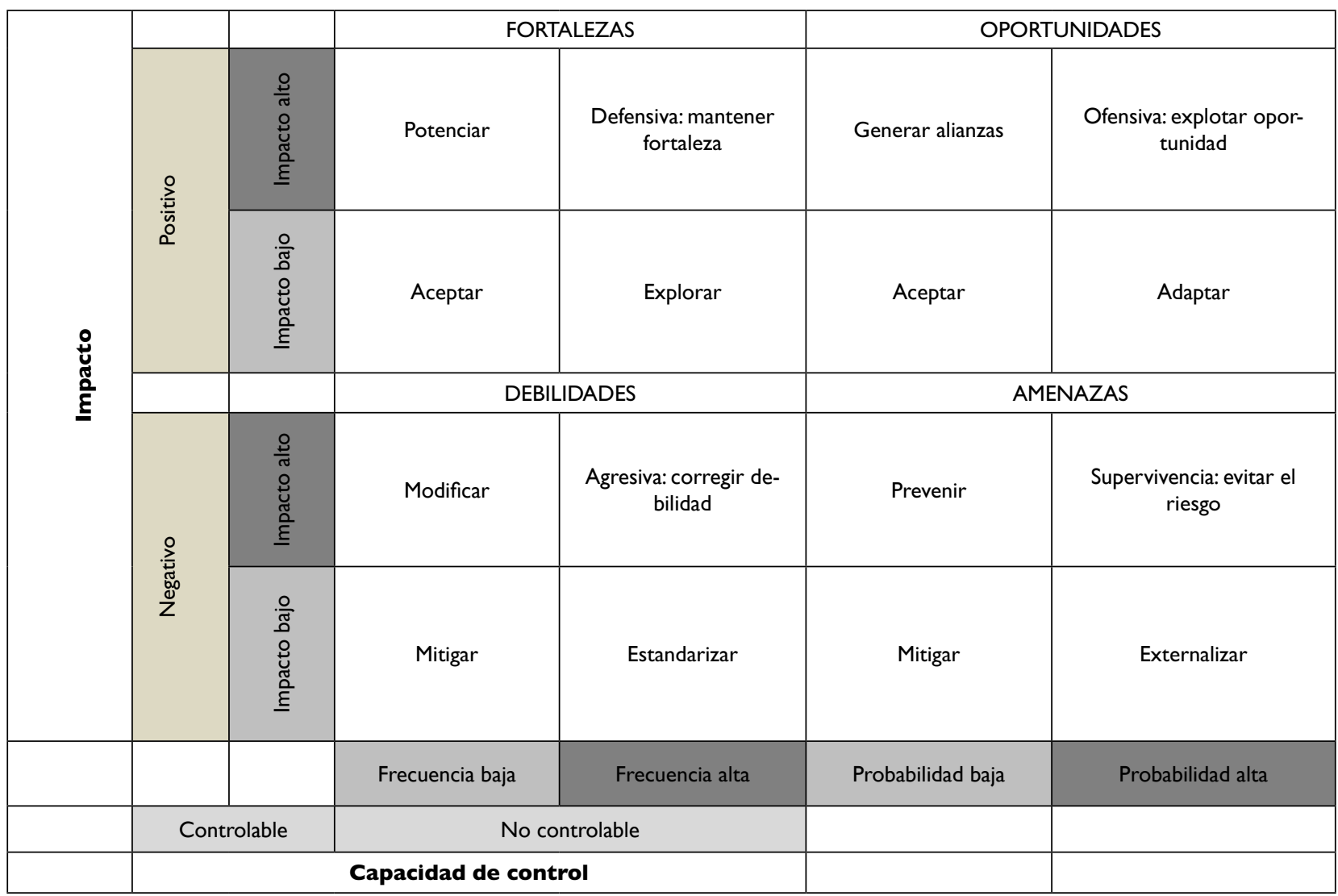

Tabla 6: Matriz de estrategias genéricas

\begin{tabular}{|l|l|}
\hline Defensiva & Es prioridad mantener el efecto positivo de este parámetro. \\
\hline Potenciar & $\begin{array}{l}\text { Es necesario encausar estrategias que potencien al parámetro como fortaleza, ya sea mejorando o ampliando su } \\
\text { efecto, tal de crear un efecto multiplicador de su impacto. }\end{array}$ \\
\hline Aceptar & Es una estrategia enfocada a aminorar esfuerzos en parámetros de bajo impacto y baja probabilidad. \\
\hline Explorar & Idear formas de aprovechamiento de estas fortaleza \\
\hline Ofensiva & Indica tomar acción frente al parámetro presentado \\
\hline Generar alianzas & Indica encausar estrategia a formas conjuntas de aprovechamiento del parámetro externo \\
\hline Adaptar & Idear propuestas de explotación de las oportunidades de manera de adaptarlas a la realidad del sistema. \\
\hline Agresiva & Es prioridad corregir debilidad, erradicar o eliminar su impacto. \\
\hline Modificar & $\begin{array}{l}\text { Estrategia más adaptativa que propone disminuir el efecto del parámetro, modificando o adaptando parte de su } \\
\text { acción o funcionamiento. }\end{array}$ \\
\hline Mitigar & $\begin{array}{l}\text { Se refiere a aceptar el efecto del parámetro pero mitigar su impacto cuando los esfuerzos de aceptarlo son } \\
\text { menores a una estrategia mayor. }\end{array}$ \\
\hline Estandarizar & Se refiere a tomar medidas estándares frente al parámetro y su impacto. \\
\hline Supervivencia & Es prioridad evitar el riesgo de cualquier forma. \\
\hline Prevenir & Se refiere a enfrentar el parámetro de forma preventiva con un plan de contingencia. \\
\hline Externalizar & $\begin{array}{l}\text { Indica enfrentar el impacto con esfuerzos tales que no modifiquen la estrategia de la organización, pudiendo } \\
\text { aplicar externalización de medidas. }\end{array}$ \\
\hline
\end{tabular}

Tabla 7: Descripción de estrategias genéricas

ISSN: 07 I8-2724. (http://www.jotmi.org)

Journal of Technology Management \& Innovation (C) Universidad Alberto Hurtado, Facultad de Economía y Negocios. 


\section{Paso 4-2: búsqueda de oportunidades basado en OTSM-TRIZ}

Tanto para parámetros en conflicto de resolución simple o factores críticos de difícil mitigación debido a las limitaciones del contexto, OTSM-TRIZ busca entregar orientaciones de carácter creativo desde una perspectiva novedosa para el habitual proceso de pensamiento estratégico, a través de la identificación de contradicciones y su búsqueda de oportunidades por medio de los principios inventivos. Una vez determinado el contexto con sus respectivos parámetros (controlables y no contrables), la Red de Contradicciones (RoC) en la cual se representan los conflictos específicos entre los parámetros permite medir y evaluar el impacto de las decisiones que pudieran tomarse en los distintos escenarios. OTSM-TRIZ distingue dos tipos de parámetros: Parámetros de control (PC), que son aquellos que pueden ser modificados en la práctica directamente, y Parámetros de evaluación (PE), aquellos que son afectados según las modificaciones de los PC.

\section{Caso Estudio: \\ Cluster Minero Región de Atacama-Chile 3.I Industria minera en Chile}

La minería en Chile juega un rol fundamental en el crecimiento económico del país; durante 2012 contribuyó en un $12 \%$ al PIB, concentró el $60 \%$ de las exportaciones nacionales, aportó un $14 \%$ de los ingresos fiscales y generó aproximadamente 700.000 empleos directos e indirectos. Con respecto al escenario futuro, Chile como poseedor del $28 \%$ de las reservas mundiales de cobre y con proyecciones de inversión que ascienden a los US\$66.167 millones en proyectos debe superar los crecientes desafíos que enfrenta la industria minera para mantener la competitividad en el largo plazo y soportar la demanda futura de mineral.

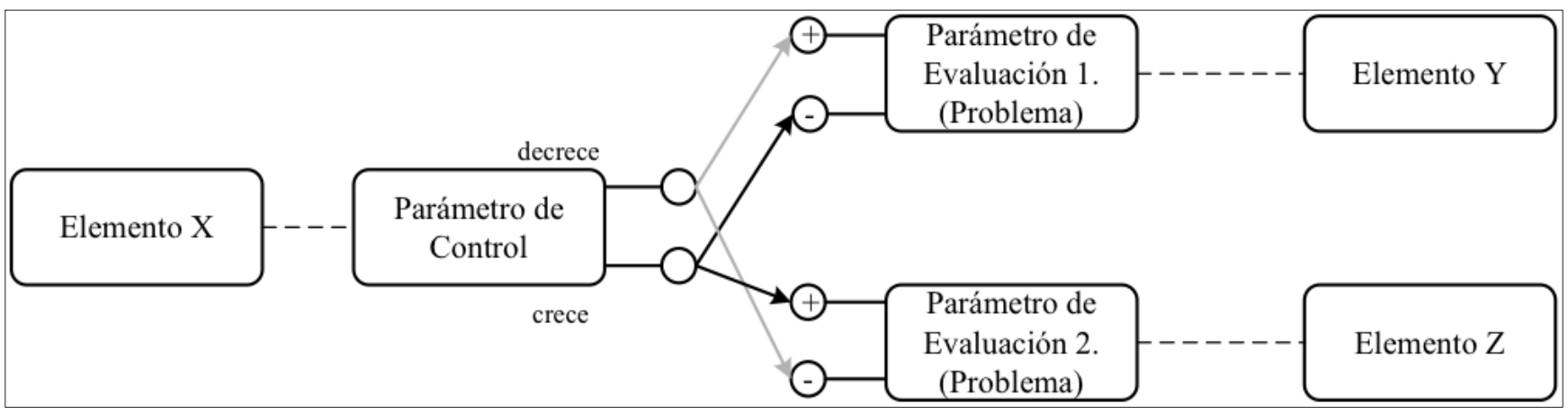

Figura 4: Esquema básico de contradicción para la Red de Contradicción en OTSM-TRIZ.

A través de la contradicción se visualizan los parámetros en conflicto y se utilizan los principios inventivos para dar solución (Altshuller, 1984).

\subsection{Clúster minero en Atacama}

La tercera región de Atacama posee una superficie de 75.452 km2 y una población estimada de 284.607 habitantes. La minería es el sector de mayor importancia en el desarrollo regional, principalmente por la explotación de cobre, oro, plata, hierro y molibdeno. En 2012 la minería alcanzó una participación de 51,3\% del Producto Interno Bruto Regional (PIBR), seguida por el sector de construcción (16,7\%). La producción de cobre fino en la región en el año 2010 tuvo una participación de $8,0 \%$ del total nacional y la producción de hierro una participación de $76,8 \%$ del total nacional; la Región de Atacama contribuyó con el $2,2 \%$ al PIB total nacional. Respecto al nivel de las exportaciones para $201 \mathrm{I}$ fue de 7.II2,8 millones de dólares, correspondiendo el 93,5\% a la minería (Instituto Nacional de Estadística, 20I2)

ISSN: 07 I8-2724. (http://www.jotmi.org) 


\subsection{Aplicación del método}

En este contexto es que se plantea la aplicación de la metodología propuesta para generar estrategias que contribuyan al desarrollo industrial de la |minería en la región de Atacama, en consideración de las problemáticas que se vinculan a su escenario, como la disminución de las leyes del mineral, los elevados costos de energía y agua y los desafíos asociados a mejorar la productividad laboral que requieren del esfuerzo conjunto de los actores participantes del sistema para encausar soluciones tanto a nivel local como global.

\section{Paso I: Caracterización del contexto actual}

El contexto del problema se ve representado por el sistema, formado por mineras y proveedores de bienes y servicios de la industria minera de la tercera región de atacama, y el entorno, que está formado por las entidades que se vinculan e interactúan con el sistema como son: entidades gubernamentales y su rol regulador, el mercado de competidores nacionales e internacionales; las universidades y centros de I+D, fundamentales para el desarrollo de conocimiento y apoyo tecnológico $y$, finalmente, la comunidad, que abarca todo ser humano y ambiente físico que, sin ser parte del proceso productivo, se ve impactado e influye de alguna forma en la actividad. Debido a que se busca generar estrategias para el desarrollo del sistema, se plantea éste como objetivo estratégico.

\section{Paso 2: Obtención de parámetros esenciales para el análisis}

Las herramientas a utilizar para la obtención de parámetros serán PESTA y cadena de valor de Porter. Con PESTA se analiza cómo afecta y beneficia el sistema al entorno y viceversa en cada una de las dimensiones de estudio: Políticolegal, Económico, Social, Tecnológico y Ambiental-energético; entregando una perspectiva macro en la que se inserta el sistema. Por otra parte, con la cadena de valor se estudian los parámetros positivos y negativos de la interacción desde el sistema hacia el mismo sistema, entre los elementos que lo conforman (mineras y proveedores) basada en el contexto del proceso productivo del mineral que relaciona a ambos elementos. Por motivos de confidencialidad del proyecto y se presenta un número reducido de parámetros para ilustrar la aplicación metodológica obtenidos durante el proceso de análisis.

\section{Paso 3: Clasificación y evaluación de parámetros}

Los parámetros obtenidos se clasifican según el tipo de impacto (positivo o negativo) y la capacidad de control que el sistema posee sobre ellos (controla $\circ$ no controla) a través de la nomenclatura antes señalada: $\mathrm{NC}-, \mathrm{NC}+, \mathrm{C}-, \mathrm{C}+$. Posteriormente, con la asignación de niveles de impacto $y$ probabilidad basados en las cifras y estadísticas que presenta la tercera región de Atacama, es posible obtener el nivel de criticidad que representa cada parámetro para el sistema y visualizar los factores negativos más críticos. Asimismo, se grafican tales parámetros según su clasificación y evaluación como se visualiza en la figura 6 .

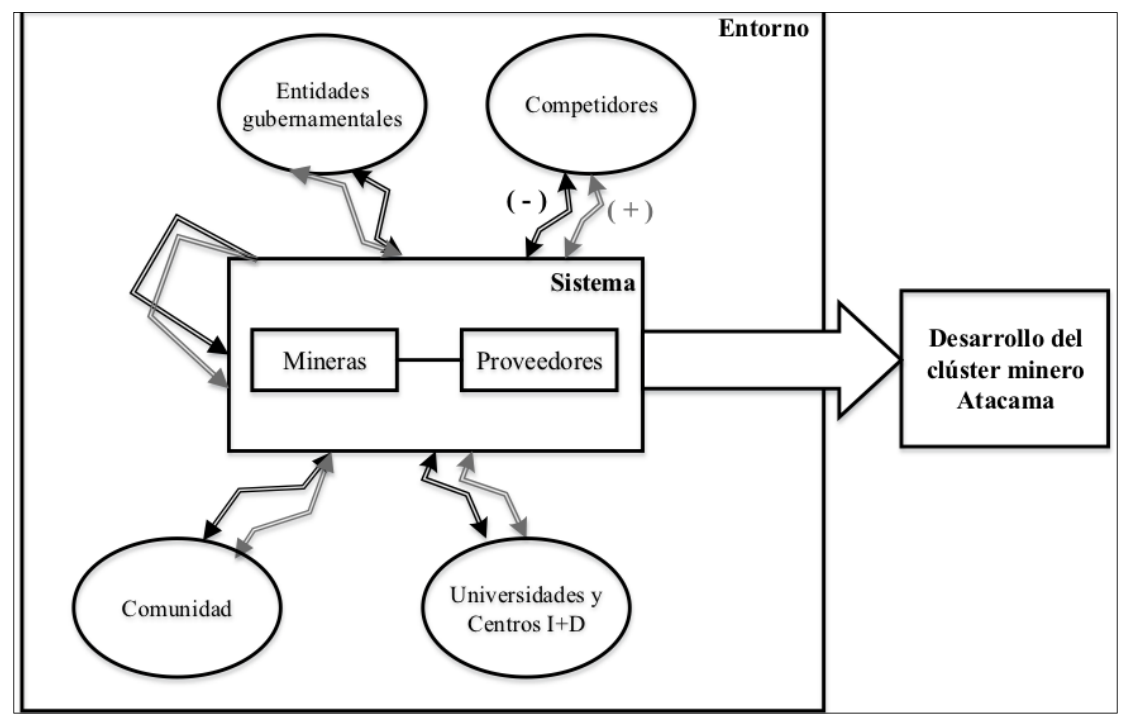

Figura 5: Modelo de interacciones del sistema Mineras-Proveedores del Clúster Minero Atacama

ISSN: 07 I8-2724. (http://www.jotmi.org)

Journal of Technology Management \& Innovation (c) Universidad Alberto Hurtado, Facultad de Economía y Negocios. 


\begin{tabular}{|c|c|c|c|c|}
\hline \multicolumn{5}{|c|}{ Obtención de Parámetros por herramienta PESTA } \\
\hline Interacción ElàE2 & Elemento I & Elemento 2 & Dimensión & Parámetro \\
\hline \multirow{3}{*}{ Interacción perjudicial Entornoà } & E. Gubernamental & Mineras & Político-legal & Aumento de impuestos \\
\hline & E. Gubernamental & Proveedores & Político-legal & $\begin{array}{l}\text { Reforma tributaria efecto } \\
\text { sobre pymes }\end{array}$ \\
\hline & Competidores & Mineras & Económico & $\begin{array}{l}\text { Competencia internacio- } \\
\text { nal mineras }\end{array}$ \\
\hline Sistema & Competidores & Proveedores & Económico & $\begin{array}{l}\text { Competencia nacional } \\
\text { proveedores }\end{array}$ \\
\hline \multirow{4}{*}{$(-)$} & Comunidad & Mineras & Social & $\begin{array}{l}\text { Mano de obra poco cali- } \\
\text { ficada/estandarizada }\end{array}$ \\
\hline & Baja ley & Mineras/Proveedores & Tecnológico & $\begin{array}{l}\text { Altos y mayores requer- } \\
\text { imientos tecnológicos }\end{array}$ \\
\hline & E. Gubernamental & Mineras/Proveedores & $\begin{array}{l}\text { Ambiental-energéti- } \\
\text { co }\end{array}$ & $\begin{array}{l}\text { Nuevas exigencias en } \\
\text { leyes medioambientales }\end{array}$ \\
\hline & E. Gubernamental & Mineras/Proveedores & $\begin{array}{l}\text { Ambiental-energéti- } \\
\text { co }\end{array}$ & $\begin{array}{l}\text { Anulación de proyectos } \\
\text { de inversión por rechazo } \\
\text { ambiental }\end{array}$ \\
\hline \multirow{4}{*}{ Interacción Benéfica Sistemaà } & Mineras & Comunidad & Económico & Contribución al PIB local \\
\hline & Mineras & Comunidad & Social & $\begin{array}{l}\text { Creación de puestos de } \\
\text { trabajo }\end{array}$ \\
\hline & Mineras & Comunidad & Social & $\begin{array}{l}\text { Polos de desarrollo en } \\
\text { zonas geográficas de ac- } \\
\text { tividad y otras industrias }\end{array}$ \\
\hline & Mineras & $\begin{array}{l}\text { Comunidad/Univer- } \\
\text { sidades/Centros de } \\
\text { I+D/Otras industrias }\end{array}$ & Tecnológico & $\begin{array}{l}\text { Introducción de nuevo } \\
\text { conocimiento tecnológi- } \\
\text { co al país }\end{array}$ \\
\hline \multicolumn{5}{|c|}{ Obtención de Parámetros por herramienta CADENA DEVALOR } \\
\hline $\begin{array}{l}\text { Interacción } \\
\text { ElàE2 }\end{array}$ & Elemento I & Elemento 2 & Dimensión & Parámetro \\
\hline \multirow[t]{6}{*}{ Interacción perjudicial Sistemaà } & Proveedores & Mineras & Abastecimiento & $\begin{array}{l}\text { Proveedores locales no } \\
\text { poseen capacidades para } \\
\text { responder a requer- } \\
\text { imientos de mineras }\end{array}$ \\
\hline & Proveedores & Mineras/Proveedores & $\mathrm{RRHH}$ & $\begin{array}{l}\text { Escasez de normas y } \\
\text { certificaciones por parte } \\
\text { de proveedores }\end{array}$ \\
\hline & Mineras/Proveedores & Mineras/Proveedores & $\begin{array}{l}\text { Capacidad tecnológi- } \\
\text { ca }\end{array}$ & $\begin{array}{l}\text { Los requerimientos de } \\
\text { capacidad tecnológica } \\
\text { son de carácter particu- } \\
\text { lar y crecientes }\end{array}$ \\
\hline & Mineras/Proveedores & Mineras/Proveedores & $\begin{array}{l}\text { Capacidad tecnológi- } \\
\text { ca }\end{array}$ & $\begin{array}{l}\text { Dependencia de tec- } \\
\text { nología extranjera }\end{array}$ \\
\hline & Mineras/Proveedores & Mineras/Proveedores & Logística interna & $\begin{array}{l}\text { Conflictos relacionales y } \\
\text { mala comunicación entre } \\
\text { proveedores y mandan- } \\
\text { tes }\end{array}$ \\
\hline & Mineras/Proveedores & Mineras/Proveedores & Operaciones & $\begin{array}{l}\text { Deficiente calidad de } \\
\text { servicios brindados por } \\
\text { proveedores }\end{array}$ \\
\hline
\end{tabular}

ISSN: 07 I8-2724. (http://www.jotmi.org) 


\begin{tabular}{|l|l|l|l|l|}
\hline \multirow{4}{*}{ Interacción Benéfica Sistemaà } & Mineras/Proveedores & Mineras/Proveedores & RRHH & $\begin{array}{l}\text { Implementación de pro- } \\
\text { gramas de capacitación } \\
\text { a proveedores por man- } \\
\text { dantes }\end{array}$ \\
\cline { 2 - 5 } Sistema & Mineras & Mineras/Proveedores & Infraestructura & $\begin{array}{l}\text { Proyectos en construc- } \\
\text { ción y futura cartera de } \\
\text { proyectos mineros }\end{array}$ \\
\cline { 2 - 5 } & Mineras/Proveedor & Mineras/Proveedores & Servicios & $\begin{array}{l}\text { Amplia gama de servicios } \\
\text { que soportan la actividad } \\
\text { principal }\end{array}$ \\
\hline
\end{tabular}

Tabla 8: Obtención de parámetros esenciales para el análisis

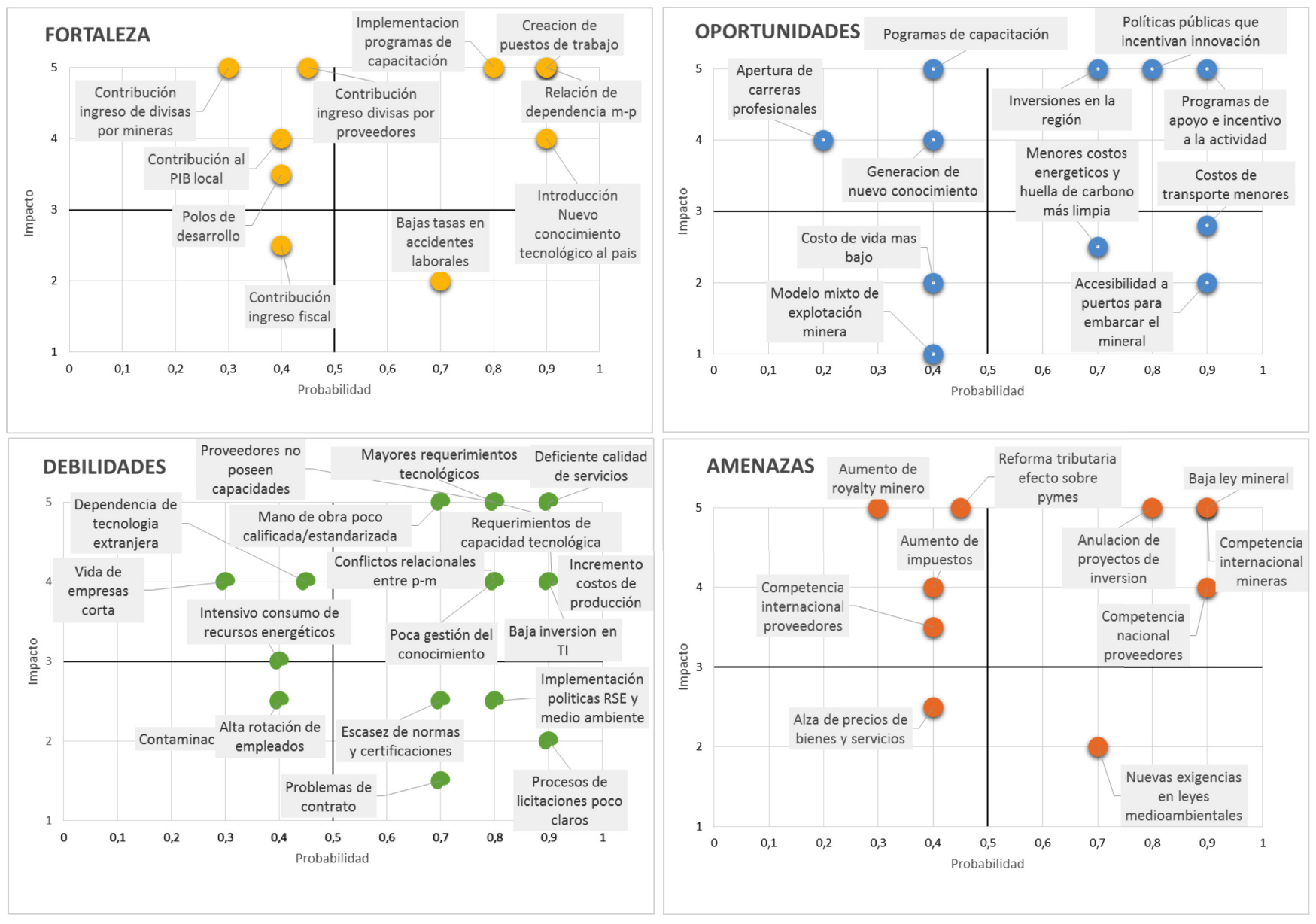

Figura 6: Gráficos de clasificación y evaluación de parámetros según niveles de impacto y eventualidad. 


\begin{tabular}{|c|c|c|c|c|c|c|c|}
\hline Parámetro & $\begin{array}{l}\text { NC } \\
-\end{array}$ & $\mathrm{NC}+$ & C - & $C+$ & Impacto & $\begin{array}{l}\text { Eventuali- } \\
\text { dad }\end{array}$ & Criticidad \\
\hline Aumento de impuestos & $x$ & & & & 4 & 0,4 & $\mathrm{I}, 6$ \\
\hline $\begin{array}{l}\text { Competencia internacional min- } \\
\text { eras }\end{array}$ & $x$ & & & & 5 & 0,9 & 4,5 \\
\hline $\begin{array}{l}\text { Competencia nacional provee- } \\
\text { dores }\end{array}$ & $x$ & & & & 4 & 0,9 & 3,6 \\
\hline $\begin{array}{l}\text { Mano de obra poco calificada/ } \\
\text { estandarizada }\end{array}$ & & & $x$ & & 5 & 0,7 & 3,5 \\
\hline $\begin{array}{l}\text { Altos y mayores requerimientos } \\
\text { tecnológicos }\end{array}$ & & & $x$ & & 5 & 0,9 & 4,5 \\
\hline Anulación proyectos de inversión & $x$ & & & & 5 & 0,8 & 4 \\
\hline $\begin{array}{l}\text { Polos de desarrollo en zona } \\
\text { geográfica y otras industrias }\end{array}$ & & & & $x$ & 5 & 0,8 & 4 \\
\hline $\begin{array}{l}\text { Introducción de conocimiento } \\
\text { tecnológico al país }\end{array}$ & & & & $x$ & 4 & 0,8 & 3,2 \\
\hline $\begin{array}{l}\text { Proveedores locales no poseen } \\
\text { capacidades para responder a } \\
\text { requerimientos de mineras }\end{array}$ & & & $x$ & & 5 & 0,8 & 4 \\
\hline $\begin{array}{l}\text { Escasez de normas y certifica- } \\
\text { ciones por parte de proveedores }\end{array}$ & & & $x$ & & 5 & 0,7 & 3,5 \\
\hline $\begin{array}{l}\text { La mayoría de proveedores no im- } \\
\text { plementa políticas de RSE y gestión } \\
\text { de medio ambiente }\end{array}$ & & & $x$ & & 2 & 0,8 & $\mathrm{I}, 6$ \\
\hline $\begin{array}{l}\text { Los requerimientos de capacidad } \\
\text { tecnológica son de carácter partic- } \\
\text { ular y crecientes }\end{array}$ & & & $x$ & & 5 & 0,8 & 4 \\
\hline $\begin{array}{l}\text { Dependencia de tecnología ex- } \\
\text { tranjera }\end{array}$ & & & $x$ & & 4 & 0,6 & 2,4 \\
\hline $\begin{array}{l}\text { Conflictos relacionales y mala } \\
\text { comunicación entre proveedores y } \\
\text { mandantes }\end{array}$ & & & $x$ & & 5 & 0,4 & 2 \\
\hline $\begin{array}{l}\text { Deficiente calidad de servicios } \\
\text { brindados por proveedores }\end{array}$ & & & $x$ & & 5 & 0,7 & 3,5 \\
\hline $\begin{array}{l}\text { Existencia de implementación de } \\
\text { programas de capacitación a prov- } \\
\text { eedores por parte de mandantes }\end{array}$ & & & & $x$ & 5 & 0,4 & 2 \\
\hline $\begin{array}{l}\text { Proyectos en construcción y futura } \\
\text { cartera de proyectos mineros }\end{array}$ & & & & $x$ & 5 & 0,8 & 4 \\
\hline $\begin{array}{l}\text { Amplia gama de servicios que } \\
\text { soportan la actividad principal }\end{array}$ & & & & $x$ & 2,5 & 0,8 & 2 \\
\hline
\end{tabular}

Tabla 9: Clasificación y evaluación de parámetros 


\section{Paso 4: Generación de estrategias propuestas en base a parámetros encontrados}

\section{Paso 4.I Matriz de estrategias genéricas}

Se ubican los parámetros en la matriz, tal que se visualicen estrategias propuestas como potenciar (I), generar alianzas (2), modificar (3), prevenir (4). En virtud de lo indicado por la matriz, el análisis estratégico propone:Proveedores y mineras deben tomar acción frente a las oportunidades y potenciar alianzas con universidades, centros I+D, entidades locales y regionales, ya que la generación de tecnología involucra financiamiento, innovación y conocimiento, además en consideración de los tiempos de ejecución y remuneración distintos entre mineras y proveedores, se requiere del apoyo de entidades públicas que propicien financiamiento. Proveedores y mineras deben corregir los efectos negativos dentro de su rango de control como las debilidades en cuánto a problemas de comunicación, coordinación y trato. Difundir concepto de ganar-ganar y cooperación en vez de rivalidad basado en las fortalezas conjuntas y en mantener lo que se ha hecho bien. Proveedores deben enfocarse en profesionalizar el servicio, mejorar presentación, capacidad de respuesta y medios de comunicación, apoyándose en herramientas de TI, innovación y gestión de conocimiento. Mineras fomentar la responsabilidad, compromiso y apoyo, para hacer frente a las amenazas de manera conjunta.

\begin{tabular}{|c|c|c|c|c|c|c|}
\hline \multirow{4}{*}{ 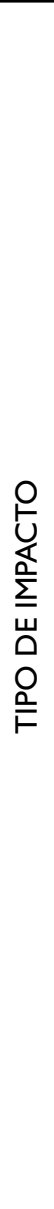 } & \multirow[t]{2}{*}{ 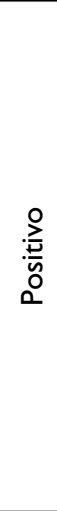 } & 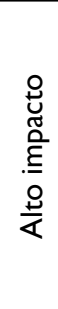 & $\begin{array}{l}\text { Implementación progra- } \\
\text { mas de capacitación a } \\
\text { proveedores (I) }\end{array}$ & $\begin{array}{l}\text { Creación de puestos de trabajo } \\
\text { Polos de desarrollo en zonas geográfi- } \\
\text { cas de actividad y para otras industrias } \\
\text { Diversificación de productos } \\
\text { Bajas tasas accidentes } \\
\text { Relación de dependencia } \\
\text { proveedores-mandantes } \\
\text { Proyectos en construcción y futuros }\end{array}$ & $\begin{array}{l}\text { Generación de } \\
\text { nuevo } \\
\text { conocimiento } \\
\text { Apertura } \\
\text { carreras } \\
\text { profesionales } \\
(2)\end{array}$ & $\begin{array}{l}\text { Políticas públicas que } \\
\text { incentivan innovación } \\
\text { Programas de apoyo e } \\
\text { incentivo a la actividad } \\
\text { Inversiones en la región } \\
\text { Programas } \\
\text { de capacitación }\end{array}$ \\
\hline & & 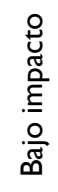 & $\begin{array}{l}\text { Contribución al PIB local } \\
\text { Contribución ingreso } \\
\text { de divisas } \\
\text { Contribución ingreso } \\
\text { fiscal }\end{array}$ & $\begin{array}{l}\text { Introducción de nuevo conocimiento } \\
\text { tecnológico al país } \\
\text { Amplia gama de servicios de soporte }\end{array}$ & $\begin{array}{l}\text { Costo de vida es } \\
\text { más bajo } \\
\text { Modelo mixto } \\
\text { de explotación } \\
\text { minera }\end{array}$ & $\begin{array}{l}\text { Accesibilidad a puertos } \\
\text { para embarcar mineral }\end{array}$ \\
\hline & \multirow[t]{2}{*}{ 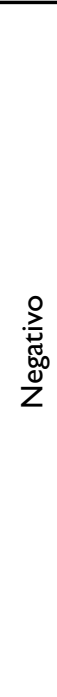 } & 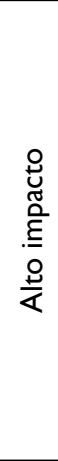 & $\begin{array}{l}\text { Conflictos relacionales y } \\
\text { mala comunicación } \\
\text { Corta vida de empresas } \\
\text { Escasez de normas y } \\
\text { certificaciones (3) }\end{array}$ & $\begin{array}{l}\text { (proveedores) } \\
\text { Mano de obra poco calificada } \\
\text { No poseen las capacidades } \\
\text { Baja inversión en TI } \\
\text { Deficiente calidad de servicios } \\
\text { (proveedores-mandantes) } \\
\text { Problemas de comunicación, } \\
\text { coordinación y desacuerdos } \\
\text { Incremento costos de producción } \\
\text { Requerimientos tecnológicos } \\
\text { específicos y creciente } \\
\text { Dependencia de tecnología extranjera }\end{array}$ & $\begin{array}{l}\text { Aumento royalty } \\
\text { minero } \\
\text { Aumento } \\
\text { impuestos } \\
\text { (4) }\end{array}$ & $\begin{array}{l}\text { Competencia nacional } \\
\text { proveedores } \\
\text { Anulación proyectos de } \\
\text { inversión } \\
\text { Reforma tributaria } \\
\text { Competencia } \\
\text { internacional mineras }\end{array}$ \\
\hline & & 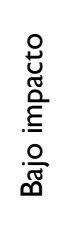 & $\begin{array}{l}\text { Contaminación } \\
\text { Intensivo consumo r. } \\
\text { energéticos }\end{array}$ & $\begin{array}{l}\text { Problemas de contrato } \\
\text { Alta rotación de empleado } \\
\text { Procesos de licitaciones poco claro } \\
\text { Escasa implementación políticas RSE y } \\
\text { gestión de medio ambiente }\end{array}$ & $\begin{array}{l}\text { Competencia } \\
\text { internacional } \\
\text { proveedores } \\
\text { Alza precios } \\
\text { bienes } \\
\text { y servicios }\end{array}$ & $\begin{array}{l}\text { Exigencias en leyes } \\
\text { medioambientales }\end{array}$ \\
\hline & & & Baja probabilidad & Alta probabilidad & Baja probabilidad & Alta probabilidad \\
\hline & & & Controlable & & \multicolumn{2}{|c|}{ No controlable } \\
\hline \multicolumn{7}{|c|}{ CAPACIDAD DE CONTROL } \\
\hline
\end{tabular}

Tabla 10: Matriz de estrategias genéricas.

ISSN: 07 I8-2724. (http://www.jotmi.org)

Journal of Technology Management \& Innovation (C) Universidad Alberto Hurtado, Facultad de Economía y Negocios. 


\section{Paso 4.2: búsqueda de oportunidades basado en OTSM-TRIZ}

A través del análisis previo se identifican los parámetros que en conjunto responden a un mismo problema, el cual se busca solucionar mediante OTSM-TRIZ. El Clúster minero propone como estrategia que las mineras mandantes prefieran la oferta de proveedores locales, lo que permite, por una parte, el crecimiento, mejora y competitividad de los proveedores, pero por otra parte, perjudica a las mineras ya que la baja calidad de bienes y servicios no se ajusta los requerimientos de su producción afectando su productividad, lo cual se presenta como una contradicción.
A modo de ejemplo de la aplicación de TRIZ como soporte al proceso de generación de estrategias, se presenta la estrategia enfocada a que "mineras prefieran a los proveedores locales" y su planteamiento como contradicción, donde se proponen tres soluciones parciales que resuelven los parámetros perjudiciales de tal contradicción:i) Generación de tecnología propia; ii) Creación de estándares de la industria; y iii) Implementación de capacitación. Las cuales se desarrollan nuevamente como contradicción y en base a ella, se aplican los principios inventivos que generan nuevas soluciones.

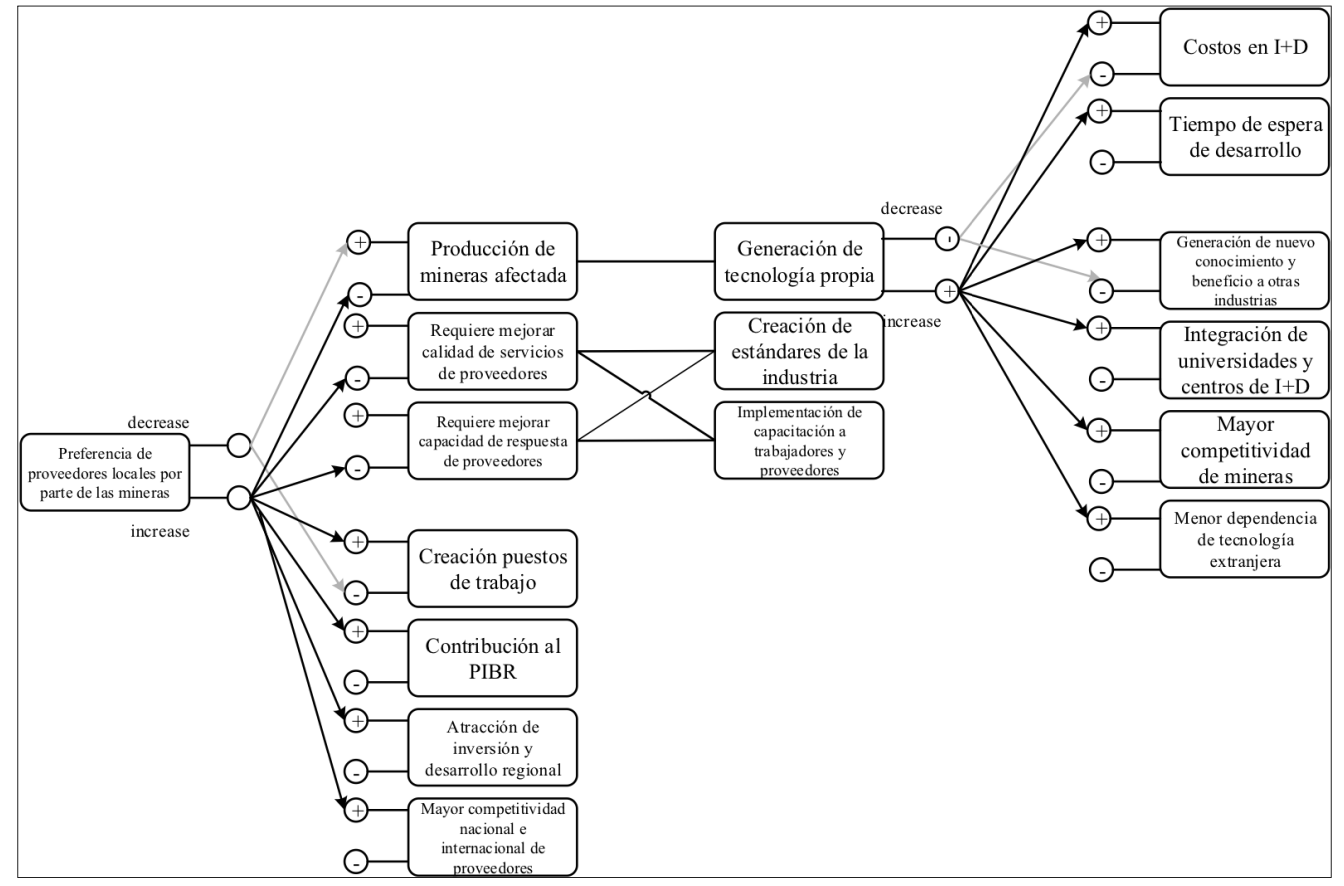

Figura 8 Gráficos de relaciones causales en base a modelo de contradicción

\begin{tabular}{|l|l|}
\hline Principios & Estrategias \\
\hline 2. Extraer, Quitar & $\begin{array}{l}\text { Incentivar investigación con universidades, centros de I+D, proveedores, entidades locales y regionales como alianza y meta } \\
\text { conjunta. } \\
\text { Separar las actividades de desarrollo y producción; producción apuntando a eficiencia, desarrollo e investigación es un pro- } \\
\text { ceso que requiere mayor tiempo y paciencia. } \\
\text { Separar la gente del problema; permitir equivocarse en cuanto a innovación se trata ya que es la única forma de crear y } \\
\text { 10.Acción previa } \\
\text { I5. Dinámica }\end{array}$ \\
$\begin{array}{l}\text { Incentivar la llegada de compañías de alta tecnología a la región de manera de propiciar la generación de tecnología e intro- } \\
\text { ducción de conocimiento beneficiando a otras industrias y al desarrollo local. } \\
\text { Involucrar a jóvenes en trabajos mediante prácticas o contrato, de manera que ingresen a la empresa nuevas ideas y mayor } \\
\text { capacidad de aprendizaje y creación de conocimiento. } \\
\text { Presentar inquietudes y aprovechar alianza y cooperación con organizaciones paraguas como CORFO, así como programas } \\
\text { de apoyo financiero para potenciar capacidades tecnológicas de proveedores. }\end{array}$
\end{tabular}

Tabla II Principios inventivos aplicados al caso de estudio (Altshuller, 1984).

ISSN: 07 I8-2724. (http://www.jotmi.org)

Journal of Technology Management \& Innovation (C) Universidad Alberto Hurtado, Facultad de Economía y Negocios. 


\section{Conclusiones}

La metodología propuesta permite orientar de manera sistémica el análisis y formulación de direcciones estratégicas, siguiendo una estructura de pasos desde la obtención de información relativa al contexto, parámetros esenciales que lo definen, además de la clasificación y evaluación de parámetros críticos hasta finalmente la generación de estrategias, integrando en su procedimiento técnicas tradicionales de análisis estratégico, nuevos instrumentos matriciales y herramientas creativas para la resolución de problemas como TRIZ y OTSM-TRIZ.

El primer paso de la metodología: la caracterización del contexto actual, a través del modelo de interacciones, es una forma distinta de planteamiento de problemas. El modelo de interacciones al ser una representación gráfica, facilita la conceptualización del contexto con un enfoque relacional, confiriendo flexibilidad al alcance y visualización de la situación. La obtención de parámetros esenciales, como segundo paso de la metodología, es un procedimiento de adquisición de información utilizando el modelo de interacciones y herramientas tradicionales. Por una parte, el modelo permite un método de estudio más amplio y completo, generando información desde distintas perspectivas, con una mirada de $360^{\circ}$, más completa y diversa del contexto; contribuyendo a la búsqueda de información y a la obtención de conocimiento, importantes principalmente para los tomadores de decisiones. La elección de métodos de estrategia está sujeta al criterio del analista, pero considerando que se debe tener en cuenta la posibilidad de estudio del sistema desde su aspecto interno y externo, motivo por el cual se entregan orientaciones en su aplicación en la tabla 3. Si bien, esto puede parecer una dificultad para inexpertos, es importante destacar que lo más relevante de este paso es generar la información tal que permita visualizar una perspectiva interna y otra externa del sistema, independientemente del tipo de herramienta que se utilice para la obtención de parámetros, ya que lo más relevante del análisis es la forma en que tal información se presenta y se ordena, como se señala en el tercer paso de la metodología: clasificación y evaluación de parámetros.

La clasificación y evaluación de parámetros, resulta ser un procedimiento que busca ordenar la información y a partir de éste y del nivel de criticidad de los parámetros, entregar nociones del grado de importancia y de su implicancia en la construcción de direcciones y determinación del tipo de estrategia a seguir. El nivel de criticidad entrega conocimiento en cuanto a las prioridades que deben tomarse en cuenta, sin embargo, se plantea como una propuesta de jerarquización, siendo igualmente válido la inclusión de parámetros críticos que por criterio propio se busque dar énfasis.
En el cuarto paso, se utilizan la matriz de estrategias genéricas y los principios inventivos de TRIZ; la primera reúne técnicas de conocimiento estratégico (FODA, matriz de probabilidad e impacto) de una forma integradora, constituyendo un instrumento matricial útil y fácil de aplicar, en un formato estructurado de organización de elementos críticos. Por su parte, el método de pensamiento por TRIZ es de gran relevancia para la generación de estrategias específicas, ya que una vez identificados los parámetros negativos del análisis, amenazas o debilidades, TRIZ permite su planteamiento como un problema y por ende seguir con la causa raíz de éste, proponiendo soluciones que atacan desde otras perspectivas. Una de las ventajas de la resolución de problemas mediante TRIZ es que, basado en la transformación de problemas particulares a problemas análogos y su vinculación a soluciones análogas para generar soluciones específicas; los principios de TRIZ resultan especialmente útiles para analistas inexpertos que no conocen de métodos de solución usuales o ya existentes frente a problemas de carácter empresarial o estratégico.

En cuanto a la combinación de la matriz de estrategias genéricas y los principios de TRIZ, es importante mencionar que si bien la primera entrega nociones generales y separadas para cada parámetro, con los principios inventivos y el método de contradicciones se logra una mirada conjunta de los parámetros críticos detectados, respondiendo con estrategias específicas para una solución conjunta.

Acorde al caso de estudio en la región de Atacama, la metodología ha generado un estudio completo e integral frente a una problemática compleja a nivel país, entregando direcciones tanto para proveedores como para mineras de una manera conjunta, lo cual radica en la mirada amplia y diversa que entrega el método logrando aprovechar las influencias de los distintos elementos en el escenario planteado como región de atacama para encausar estrategias que potencian el desarrollo del sector. La metodología ha sido evaluada positivamente por la CORF-Chilena por los siguientes motivos: i) permite ser de fácil utilización al menos para detectar el sistema y elementos que interactúan, además de ser extrapolable a todo tipo de ámbitos, ya sea sociales, tecnológicos, económicos y medio ambientales: ii) Permite identificar los parámetros críticos dentro del proceso de generación de estrategias pero sin perder algún aspecto relevante dada la visión holística de la metodología: iii) la propuesta basada en TRIZ permite generar una estrategia más efectiva hacia donde deben apuntar las direcciones de cambio, además considera los nexos causales entre parámetros. No obstante algunas limitaciones emergieron durante la aplicación, por ejemplo los niveles de impacto y probabilidad planteados para la clasificación y evaluación de parámetros en la metodología, son en virtud de las particularidades y preferencias de la organización por lo cual la relevancia en 
tiempo puede ir cambiando. Consecuentemente, un monitoreo constante de estos parámetros es importante para asegurar la efectividad de estrategias a largo plazo.

\section{Recomendaciones y futuros trabajos}

En cuanto a las herramientas usadas para estudiar las interacciones entre elementos, es importante destacar que todas son integrables al modelo y ya que son ampliamente conocidas, no deberían presentar mayor dificultad a la hora de aplicarlas.Además de las utilizadas en el caso estudio, existen otras herramientas como: Modelo de las 7S de McKenzie, que entrega las siguientes dimensiones de estudio: style (estilo o cultura), staff (personal), systems, strategy, strucure, skills y shared values; Modelo de las 5 fuerzas de Porter, que confiere dimensiones de estudio del ámbito más competitivo como: amenaza de entrada de nuevos competidores, poder de negociación de proveedores, poder de negociación de compradores, amenaza de ingreso de productos sustitutos y rivalidad entre los competidores; Análisis VRIO, entre otros. Finalmente, en virtud de las características integradoras de la herramienta, futuros desarrollos apuntan a la aplicación del método a la generación de estrategias en el ámbito público y de la política industrial, contribuyendo a la generación de direcciones para resolver problemas complejos a nivel macro.

Adicionalmente, los autores proponen este modelo para crear una plataforma de análisis que permita hacer propuestas de estrategias. El objetivo es generar análisis estratégicos más completos además de apoyar el proceso de análisis para expertos e inexpertos.

\section{Agradecimientos}

Proyecto Cluster Minero financiado por la corporación de fomento de la producción (CORFO). Numero de proyecto 08-CMOI-I4.Al grupo de trabajo que del Centro Científico Tecnológico de Valparaíso (CCTVal) por el apoyo en este proyecto.

\section{Referencias}

ALTSHULLER, G. S. (1984). Creativity as an exact science. Gordon and Breach, Science Publishing, New York.

ALTSHULLER, G., Shulyak, L., \& Rodman, S. (1999). The innovation algorithm:TRIZ, systematic innovation and technical creativity. Technical Innovation Center, Inc.

BECATTINI, N., \& Cascini, G. (20I3). Mapping Causal Relationships and Conflicts among Design Parameters and System Requirements. Computer-Aided Design and Applications, I0(4), 643-662. Doi: 10.3722/cadaps.2013.643-662

BECATTINI, N., \& Cascini, G. (20/4). General-purpose requirements checklist for improving the completeness of a design specification. In DS 77: Proceedings of the DESIGN 2014 13th International Design Conference

BORREGO,A.A., Barrantes, C. L., \& Boza, O. C. (20I0). Modelo de análisis y formulación estratégica. Empleando herramientas matriciales. Industrial Data, I3(I), 009-0I7.

CAVALLUCCI, D., \& Khomenko, N. (2006). From TRIZ to OTSM-TRIZ: addressing complexity challenges in inventive design. International Journal of Product Development, 4(I2), 4-2I. DOI: I0.1504/IJPD.2007.0II 530

DAVID, F. R. (2003). Conceptos de administración estratégica. Pearson Educación.

DYSON, R. G. (2004). Strategic development and SWOT analysis at the University of Warwick. European journal of operational research, I52(3), 63I-640. DOI: I0.1016/S03772217(03)00062-6

DUNCAN,W.R. (1996).A guide to the project management body of knowledge.Ed. Project Management Institute, Inc.

HARRIS, L. C., \& Ogbonna, E. (2006). Initiating strategic planning. Journal of Business Research, 59(I), I00-I I I. DOI: 10.1016/j.jbusres.2005.02.003

HILL, T., \& Westbrook, R. (1997). SWOT analysis: it's time for a product recall. Long range planning, 30(I), 46-52. DOI: I0.1016/S0024-630I (96)00095-7

HOUBEN, G., Lenie, K., \& Vanhoof, K. (1999). A knowledgebased SWOT-analysis system as an instrument for strategic planning in small and medium sized enterprises. Decision support systems, 26(2), 125-135. DOI: 10.1016/S01679236(99)00024-X 
INSTITUTO NACIONAL DE ESTADISTICA (20I2). Compendio estadístico 2012 Síntesis geográfica nacional. Revisado en: www.ine.cl/

JIMÉNEZ,A. C. (20II). Deficiencias en el uso del foda causas y sugerencias.Revista Ciencias Estratégicas, 19(25), 89-100

JOHNSON, G., Scholes, K., Whittington, R., López, Y. M., \& Mazagatos, V. B. (200I). Dirección estratégica (Vol. 5). Prentice Hall.

KANGAS, J., Kurttila, M., Kajanus, M., \& Kangas, A. (2003). Evaluating the management strategies of a forestland estate- the SOS approach. Journal of environmental management, 69(4), 349-358.DOI: 10.1016/j.jenvman.2003.09.010 .

KHOMENKO, N., De Guio, R., Lelait, L., \& Kaikov, I. (2007).A framework for OTSM? TRIZ-based computer support to be used in complex problem management. International journal of Computer Applications in Technology,30 (I-2), 88-104. DOI: I0.1504/IJCAT.2007.0I5700

KLINGER, B. (2006). Uncertainty in the search for new exports. Center for International Development at Harvard University.

KOONTZ, H., \& Weihrich, H. (1998). Administración: una perspectiva global (Vol. I I). McGraw-Hill.

MARIÑO Ibáñez,A., Cortés Aldana, F.A., \& Garzón Ruiz, L.A. (2008). Herramienta de software para la enseñanza y entrenamiento en la construcción de la matriz DOFA. Ingeniería e Investigación, 28(3), 159-164.

MINTZBERG, H., Quinn, J. B., \& Voyer,J. (1997). El proceso estratégico: conceptos, contextos y casos. Pearson Educación.

NIKULIN, C., Graziosi, S., Cascini, G., Araneda,A., \& Minutolo, M. (20I3). An algorithm for supply chain integration based on OTSM-TRIZ. Procedia-Social and Behavioral Sciences, 75, 383-396. DOI: 10.1016/j.sbspro.2013.04.043

PHILIP, K. (1994). Marketing management: analysis planning implementation and control. Pearson Education (US); Edición: 8th Revised edition (22 de octubre de 1993)

RUCHTI, B., \& Livotov, P. (200I). TRIZ-based innovation principles and a process for problem solving in business and management. The TRIZ Journal, I, 677-687.

SAVRANSKY, S. (2000), Engineering of Creativity: Introduction to TRIZ Methodology of Inventive Problem Solving. CRC Press, Florida.
STEWART, R.A., Mohamed, S., \& Daet, R. (2002). Strategic implementation of IT/IS projects in construction: a case study. Automation in Construction, II(6), 68I-694.DOI: 10.1016/ S0926-5805(02)00009-2

THOMPSON, J.,Arthur,A., \& Strickland III,A.J. (1994). Dirección y administración estratégicas: conceptos, casos y lecturas. Adison Wesley

VIVEROS, P., Zio, E., Nikulin, C., Stegmaier, R., \& Bravo, G. (20I4). Resolving equipment failure causes by root cause analysis and theory of inventive problem solving. Proceedings of the Institution of Mechanical Engineers, Part O: Journal of Risk and Reliability, 228(I), 93-III. DOI: $10.1177 / 1748006 \times 13494775$ 